\title{
Avaliação do perfil lipídico de pacientes diabéticos e hipertensos tratados com captopril
}

\section{Evaluation of lipid profile in diabetic and hypertensive patients treated with captopryl}

Fernanda Bernardes Fernandes Santos'; Estela Silva Balzaneli'; Márcia Regina Pessoa D’Andrade²

\section{unitermos resumo}

Perfil lipídico

Diabetes mellitus tipo 2

Hipertensão arterial

Captopril
Introdução e justificativa: Doença arterial coronariana (DAC) é a principal causa mundial de morte em indivíduos adultos, e como importantes fatores de risco tratáveis envolvidos estão o diabetes mellitus (DM), as dislipidemias e a hipertensão arterial sistêmica (HAS). O tratamento com anti-hipertensivos pode provocar alterações indesejáveis no perfil lipídico, atenuando seus efeitos benéficos antiaterogênicos na redução da pressão arterial (PA). Objetivo: Avaliar o perfil lipídico de indivíduos diabéticos tipo 2 com hipertensão essencial tratados somente com captopril ou em combinação com outros anti-hipertensivos, procurando evidenciar a melhora no padrão lipídico destes pacientes, levando a efeito protetor. Material e métodos: Foram avaliados níveis de colesterol total (CT), colesterol da lipoproteína de alta densidade (HDL-C), colesterol da lipoproteína de baixa densidade (LDL-C) e triglicérides (TG) de 140 pacientes encaminhados ao laboratório de análises clínicas da Universidade do Oeste Paulista (UNOESTE), com idade média de 59,7 $\pm 10,9$ anos, de ambos os sexos, portadores de diabetes tipo 2 e hipertensão. O controle glicêmico foi determinado pela dosagem de hemoglobina glicada $(\mathrm{HG})$. Estes pacientes foram subdivididos de acordo com controle metabólico glicêmico (satisfatório ou comprometido) em dois grupos: tratados somente com captopril (DC) e tratados com combinação captopril e hidroclorotiazida (HCTZ). Dos pacientes com HG < 8,8\% (controlado), DC e HCTZ apresentaram respectivamente níveis de CT (210,2 $\pm 43,7$ e 198,3 $\pm 37,5)$, HDL $(49 \pm 10,9$ e 49,2 $\pm 8,7), \operatorname{LDL}(126,9 \pm 43,3$ e 119,8 $\pm 23,8)$, TG $(181,9 \pm 86,4$ e 161,2 $\pm 83,8)$ e G $(151,3$ $\pm 51,4$ e $163 \pm 66,5)$, não havendo diferenças significativas entre eles. Já nos pacientes com HG > 11,2\% (não-controlado), DC e HCTZ obtiveram respectivamente níveis de CT (214,3 $\pm 45,9$ e $197 \pm 49,5)$, HDL $(35,3 \pm 20,5$ e $41,5 \pm 0,7)$, LDL $(121 \pm 43,3$ e $116,5 \pm 38,9)$, TG $(271,5 \pm 175,2$ e $194 \pm 49,5)$ e $G(232,3$ $\pm 102,9$ e $272 \pm 53,7)$, também não havendo diferenças significativas. No entanto, observa-se que, nestes últimos, a combinação do captopril com hidroclorotiazida levou a menores níveis de TG e níveis de HDL ligeiramente elevados, indicando que a associação tem efeito protetor, diminuindo o risco ateroesclerótico desses pacientes e minimizando o efeito deletério aterogênico da HCTZ.

\section{abstract}

Introduction and background: Coronary artery disease $(C A D)$ is the leading cause of death in adults worldwide and its most important treatable risk factors are diabetes mellitus (DM), dyslipidemia and systemic hypertension (SH). The treatment with anti-hypertensives may cause undesirable changes in lipid profile, which diminishes its beneficial anti-atherogenic effects on the reduction of blood pressure. Objective: To evaluate the lipid profile of type 2 diabetic subjects with essential hypertension treated only with captopryl or in combination with other anti-hypertensive drugs in order to show improvement in the lipid pattern of these patients, which leads to a protective effect. Material and methods: We assessed total cholesterol (TC), HDL cholesterol (HDL-C), LDL cholesterol ( $L D L-C)$ and triglycerides (TG) levels of 140 patients, average age $59.7 \pm 10.9$, of both sexes, with type 2 diabetes and hypertension, referred to UNOESTE Laboratory of Clinical analysis. The glycemic control was determined by glycated hemoglobin (HG) levels. According to metabolic glycemic control (satisfactory or unsatisfactory), these patients were divided into two groups: treated only with captopril (DC); treated with a combination of hydrochlorothiazide (HCTZ) and DC. In patients with $\mathrm{GH}<8.8 \%$ (controlled), DC and HCZT showed the following levels respectively: total cholesterol (210.2 \pm 43.7 and 198.3 \pm 37.5$)$, HDL (49 \pm 10.9 and 49.2 \pm 8.7$), L D L(126.9 \pm 43.3$ and $119.8 \pm 23.8), T C(181.9 \pm 86.4$ and $161.2 \pm 83.8)$ and $G(151.3$ \pm 51.4 and $163 \pm 66.5$ ). There were no significant differences among them. In patients with $\mathrm{GH}>11.2 \%$ (uncontrolled), DC and HCTZ showed the following levels respectively: total cholesterol (214.3 \pm 45.9 and 197 $\pm 49.5), \operatorname{HDL}(35.3 \pm 20.5$ and $41.5 \pm 0.7), \operatorname{LL}(121 \pm 43.3$ and $116.5 \pm 38.9), T C(271.5 \pm 175.2$ and 194 $\pm 49.5)$ and $\mathrm{G}(232.3 \pm 102.9$ and $272 \pm 53.7)$. There were no significant differences, either. Nonetheless, it was observed in the last group, the combination of captopryl with HCTZ led to lower levels of TG and slightly elevated HDL levels, which shows that the association has a protective effect by reducing the atherosclerotic risk and minimizing the deleterious atherogenic effects of HCTZ. key words

Lipid profile

Type 2 diabetes mellitus

Hypertension

Captopril 


\section{Introdução}

Diabetes mellitus (DM) é um distúrbio que acomete aproximadamente 150 milhões de pessoas no mundo e aproximadamente $90 \%$ dos casos de DM são do tipo 2 (DM2) ${ }^{(11,24)}$. As complicações em longo prazo devido a alterações micro e macrovasculares que levam a disfunção, dano ou falência de vários órgãos induzem os diabéticos a apresentarem elevado risco de doença vascular aterosclerótica, como as doenças coronarianas, arterial periférica e vascular cerebral(3), que são as principais responsáveis pela morbidade e mortalidade e representam a principal causa de morte (52\%) em pacientes $\mathrm{DM}^{(2)}$. Os estudos clínicos, Diabetes Control and Complications Trial (DCCT) (1993) e o United Kingdom Prospective Diabetes Study (UKPDS) (1998)(22) concluíram que o risco dessas complicações é diretamente proporcional à hiperglicemia persistente e a estratégia de prevenção baseia-se em um controle satisfatório que implica em controle metabólico terapêutico e de acompanhamento diagnóstico atualmente, por meio da hemoglobina glicada (HbA1c), teste mais indicado na quantificação do risco(20).

Hipertensão arterial sistêmica (HAS) afeta cerca de um bilhão de pessoas no mundo, constituindo um fator de risco independente para a doença cardiovascular (DCV) ${ }^{(4)}$. Além disso, acomete cerca de $70 \%$ dos pacientes com $\mathrm{DM}^{(8)}$. A morte de $80 \%$ dos diabéticos dá-se em decorrência de eventos trombóticos associados com HAS e dislipidemia( ${ }^{(3)}$. É de extrema importância reconhecer alterações do perfil lipídico, principalmente quando a patogênese multifatorial do diabetes pode-se agravar pela HAS e ambas estão associadas à resistência insulínica ${ }^{(7,9)}$, contribuindo para aumentar o risco da $\mathrm{DCV}^{(12)}$. Por outro lado, a maioria dos estudos prospectivos de tratamento de HAS não inclui pacientes com diabetes e as decisões são extrapoladas de resultados obtidos em pacientes não-diabéticos ${ }^{(13)}$.

Atualmente, mesmo existindo uma variedade de drogas anti-hipertensivas promovendo satisfatória redução da pressão sanguínea, várias ainda produzem efeitos colaterais adversos. As V Diretrizes Brasileiras de Hipertensão(23) enfatizam o uso das maiores classes de drogas anti-hipertensivas, como diuréticos, inibidores adrenérgicos, bloqueadores de canal de cálcio, inibidores da enzima conversora da angiotensina (IECA), bloqueadores dos receptores de AT1 da angiotensina II e vasodilatadores direto, em que o $6^{\text {th }}$ Joint National Committee on the Treatment, Detection and Follow-up of High Blood Pressure prefere iniciar o tratamento anti-hipertensivo em casos não complicados com betabloqueadores ou diuréticos ${ }^{(5)}$. No entanto, essas drogas apesar de produzirem redução da pressão sanguínea têm menor benefício na proteção da doença cardíaca coronariana (DCC).

Os efeitos dos anti-hipertensivos no perfil lipídico variam tanto na classe farmacológica, quanto no fármaco especificamente. Diuréticos tiazídicos, como hidroclorotiazida (HCTZ), clortalidona e betabloqueadores não-cardiosseletivos e sem atividade simpaticomimética intrínseca, como o propranolol, são os principais agentes deletérios ao perfil lipídico ${ }^{(16)}$. Os diuréticos tiazídicos e de alça aumentam os níveis de colesterol total (CT), lipoproteína de baixa densidade (LDL) e colesterol da lipoproteína de muito baixa densidade (VLDL-C), enquanto a lipoproteína de alta densidade (HDL) permanece inalterada ${ }^{(3,5)}$. Betabloqueador reduz HDL moderadamente e induz a aumento de triglicerídeos ${ }^{(5)}$, aumentando o fator de risco, contribuindo para a progressão da aterosclerose e reduzindo o benefício do tratamento anti-hipertensivo.

Por outro lado, os IECAs, como o captopril, parecem ter efeito neutro no perfil lipídico, ou mesmo melhorá-lo em hipertensos hipercolesterolêmicos, por meio de redução dos valores de $\mathrm{LDL}$ colesterol e dos níveis de $\mathrm{CT}^{(1,10)}$. Além disso, IECA tem sido muito recomendado a pacientes DM2, por apresentar efeito benéfico na sensibilidade insulínica ${ }^{(19)}$, no entanto, mais de $50 \%$ dos pacientes $\mathrm{DM} 2$ requerem uma segunda droga para controle pressórico efetivo ${ }^{(21)}$.

Portanto, anti-hipertensivos devem, além de melhorar o controle pressórico, não alterar o perfil lipídico e o controle glicêmico em indivíduos diabéticos, para assim atuar como redutor de eventos aterotrombóticos ${ }^{(1)}$. Com isso, o presente trabalho tem como objetivo determinar possíveis alterações do perfil lipídico em pacientes DM2 com hipertensão essencial tratados somente com captopril ou em combinação com outros anti-hipertensivos, procurando evidenciar a melhora no padrão lipídico, levando a efeito protetor, visto que os efeitos dos anti-hipertensivos no metabolismo lipídico têm sido pouco investigado.

\section{Material e métodos}

Foram coletados dados de 140 pacientes, de ambos os sexos, com idade média de 59,7 $\pm 10,9$ anos, portadores de DM2 e hipertensos em tratamento, que foram atendidos regularmente nas Unidades Básicas de Saúde (UBS) de Presidente Prudente, São Paulo, e encaminhados pela equipe médica ao Laboratório de Análises Clínicas para realização de exames para controle glicêmico por meio 
da dosagem da HbA1c, glicemia e perfil lipídico selecionados por critérios clínicos e laboratoriais. Classificados como hipertensos essenciais os indivíduos fazendo uso de medicação anti-hipertensiva, foram excluídos dados de pacientes com doenças renal, hepática ou tireoidiana e aqueles que receberam terapia de reposição hormonal ou terapia hipolipemiante. Com relação ao tratamento antihipertensivo, não se considerou a posologia ou o tempo de uso do tratamento, e os pacientes foram subdivididos em grupos inicialmente de acordo com a terapêutica empregada: (A) hipertensos em uso de monoterapia com captopril; (B) diabéticos e hipertensos em uso de monoterapia com captopril; (C) diabéticos e hipertensos tratados com captopril associado com diurético tiazídico; (D) diabéticos e hipertensos tratados com captopril associado a outros anti-hipertensivos; (E) diabéticos e hipertensos tratados com outros anti-hipertensivos sem captopril; e posteriormente, foram subdivididos em dois grupos, de acordo com o estado de controle glicêmico: diabéticos hipertensos com controle metabólico glicídico satisfatório ou comprometido, mas tratados somente com captopril (DC) e diabéticos hipertensos com controle metabólico glicídico satisfatório e comprometidos tratados com captopril associado à HCTZ. Os dados demográficos dos participantes estão representados na Tabela 1.

As amostras de sangue venoso foram obtidas com o paciente em jejum de 12 horas. As dosagens de $\mathrm{HbA1c}$ foram determinadas pela técnica de microcromatografia em minicolunas contendo resina de troca iônica, baseado na diferença na carga iônica, método de rotina disponível comercialmente para a realização do teste de $\mathrm{HbA1c}$ em laboratórios de pequeno e médio portes. No entanto, não é certificado pelo National Glycohemoglobin Standardization Program (NGSP), apresenta um valor de referência de 5,3 a $8 \%$ para população em geral e para diabéticos o método sugere controle glicêmico ótimo, valores menores que $8,8 \%$. De acordo o Posicionamento Oficial 2004 do Grupo Interdisciplinar de Padronização da $\mathrm{HbA1} \mathrm{c}^{(20)}$, o valor de meta para os resultados obtidos por métodos não-certificados pelo NGSP deve ser o resultado do limite superior do valor de referência mais uma unidade, ou seja, como o valor superior de referência do método empregado no estudo é de $8 \%$, a soma de mais uma unidade é igual a 9\%. É importante ressaltar que esse valor não é considerado de referência, mas somente utilizado como meta de controle metabólico desejável, acreditando-se que pacientes com valores abaixo de $9 \%$ terão menor risco de desenvolvimento de complicações da doença. O método usado sugere como controle ótimo o valor menor que $8,8 \%$.

As dosagens de colesterol total, triglicerídeos e glicose foram determinadas pelo método enzimático colorimétrico. O HDL-colesterol foi quantificado pelo método direto enzimático homogêneo. O método de cálculo de Friedewald ou método direto, quando necessário, foi empregado para as determinações de LDL-colesterol.

$\mathrm{Na}$ análise estatística, com apoio do programa GraphPAD 3.0, o teste de análise de variância (ANOVA) com pós-teste pelo método Student-Newman-Keuls foi utilizado para comparar os parâmetros colesterol total, HDL-colesterol, LDL-colesterol, triglicerídeos, glicose e hemoglobina glicada. Foi também realizado teste $t$ de Student, pareado, para comparar os resultados do perfil lipídico e glicídico dos diabéticos com controle metabólico adequado e comprometido. Valor de $p<0,05$ foi considerado estatisticamente significativo.

\section{Resultados}

Os resultados coletados das análises bioquímicas realizadas estão demonstrados na Tabela 2. Foram observadas diferenças estatísticas para $\mathrm{G}$ e HbA1c entre a média do grupo $A$ com relação às médias dos demais grupos $(p<0,05)$. Para CT, HDL, LDL e TG não houve diferenças significativas entre as médias dos grupos. As Figuras 1 e $\mathbf{2}$ relatam os valores médios e desvio padrão só dos pacientes diabéticos, subdivididos de acordo com o estado de controle glicêmico. Os pacientes denominados DC são diabéticos hipertensos tratados somente com captopril e os HCTZ são pacientes diabéticos hipertensos tratados com captopril e hidroclorotiazida. Na Figura 1 os pacientes encontram-se em controle glicêmico satisfatório de acordo com os valores encontrados da $\mathrm{HG}$, ou seja, $\mathrm{HG}<8,8 \%$, e não se observam diferenças significativas nos níveis de CT, HDL, LDL, TG e G entre os dois grupos. Na Figura 2, os pacientes estão com controle metabólico comprometido, ou seja, HbA1c > 11,2\%, e também não foram observadas diferenças significativas entre o perfil lipídico e a glicemia entre os grupos DC e HCTZ. No entanto, o grupo HCTZ apresentou níveis de TG menores que o grupo DC, mesmo apresentando níveis glicêmicos maiores. Quanto aos valores de HDL, foram relativamente semelhantes, em que os pacientes HCTZ apresentaram desvio padrão muito pequeno à escala do gráfico apresentado. 
Caracterização dos grupos estudados

Tabela 1 expressos em média e desvio padrão

\begin{tabular}{lccccc}
\hline & $(\mathrm{A})$ & $(\mathrm{B})$ & $(\mathrm{C})$ & $(\mathrm{D})$ & $(\mathrm{E})$ \\
$n(\mathrm{M} / \mathrm{F})$ & 32 & 37 & 14 & 24 & 33 \\
& $(10 / 22)$ & $(15 / 22)$ & $(05 / 09)$ & $(04 / 20)$ & $(07 / 26)$ \\
Idade & $61,1 \pm$ & $60,8 \pm$ & 60,5 & $57,2 \pm$ & 61,8 \\
(anos) & 13 & 11,7 & \pm 9 & 12,2 & $\pm 9,6$
\end{tabular}

n: tamanho; M: sexo masculino; F: sexo feminino; (A): hipertensos em uso de monoterapia com captopril; (B): diabéticos e hipertensos em uso de monoterapia com captopril; (C): diabéticos e hipertensos tratados com captopril associado com hidroclorotizídicos; (D): diabéticos e hipertensos tratados com captopril associado a outros anti-hipertensivos; (E):

diabéticos e hipertensos tratados outros anti-hipertensivos sem captopril.

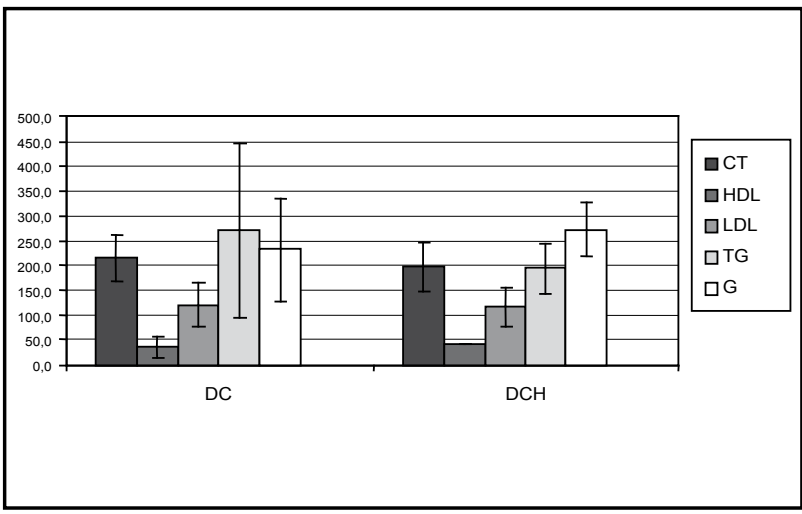

Figura 2 - Pesquisa da mutação G20210A da protrombina no paciente com trombose venosa profunda e em seus pais. Raia 1: marcador de peso molecular; raia 2: mãe; raia 3: pai. raia 4: paciente, raia 5: controle-negativo; raia 6: não-digerido; raia 7: água Milli Q

CT: colesterol total; HDL: lipoproteína de alta densidade; LDL: lipoproteína de baixa densidade; TG: triglicerídeos; C: glicose.

\section{Tabela 2 Dados coletados dos exames laboratoriais dos grupos em estudo}

\begin{tabular}{|c|c|c|c|c|c|c|}
\hline & (A) & (B) & (C) & (D) & (E) & Valor de $p$ \\
\hline CT (mg/dl) & $216 \pm 13$ & $209 \pm 40$ & $195 \pm 42$ & $197 \pm 41$ & $200 \pm 50$ & NS \\
\hline $\mathrm{HDL}(\mathrm{mg} / \mathrm{dl})$ & $51 \pm 13$ & $47 \pm 14$ & $51 \pm 9$ & $47 \pm 12$ & $49 \pm 12$ & NS \\
\hline LDL (mg/dl) & $132 \pm 40$ & $124 \pm 38$ & $112 \pm 29$ & $114 \pm 30$ & $112 \pm 40$ & NS \\
\hline $\mathrm{TG}(\mathrm{mg} / \mathrm{dl})$ & $170 \pm 71$ & $198 \pm 116$ & $167 \pm 85$ & $198 \pm 89$ & $193 \pm 112$ & NS \\
\hline $\mathrm{G}(\mathrm{mg} / \mathrm{dl})$ & $109 \pm 12^{(*)}$ & $178 \pm 61$ & $185 \pm 73$ & $191 \pm 92$ & $177 \pm 58$ & $<0,05$ \\
\hline $\mathrm{HbAlc}(\%)$ & $7,4 \pm 1,3^{(*)}$ & $9,0 \pm 1,6$ & $9,0 \pm 1,8$ & $9,5 \pm 2,3$ & $9,0 \pm 2,1$ & $<0,05$ \\
\hline
\end{tabular}

* Dados representados pelas médias e desvio padrão de CT, HDL, LDL, TG e HbAlc.

CT: colesterol total; HDL: lipoproteína de alta densidade; LDL: lipoproteína de baixa densidade; TG: triglicerídeos; G: glicose; HbAlc: hemoglobina glicada; NS = diferenças não significativas; (A): hipertensos em uso de monoterapia com captopril; (B): diabéticos e hipertensos em uso de monoterapia com captopril; (C): diabéticos e hipertensos tratados com captopril associado com hidroclorotizídicos; (D): diabéticos e hipertensos tratados com captopril associado a outros anti-hipertensivos; (E): diabéticos e hipertensos tratados outros anti-hipertensivos sem captopril.

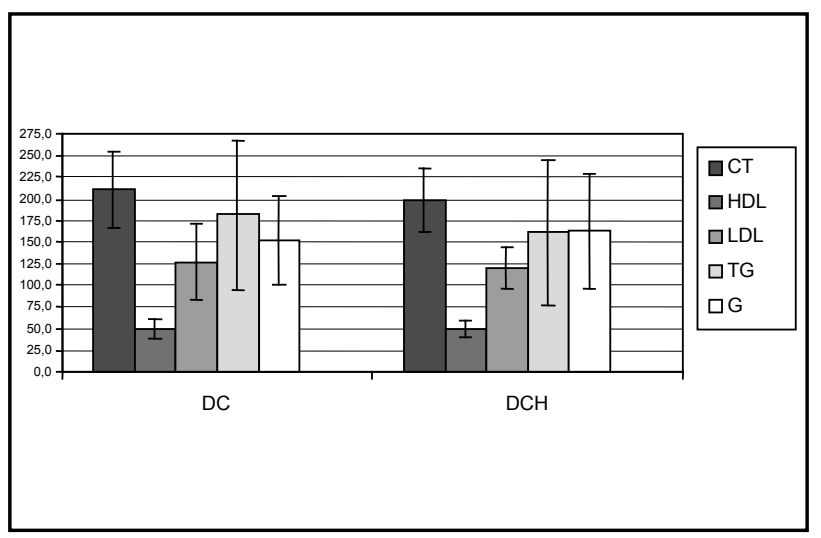

Figura 1 - Pesquisa do fator V de Leiden na criança e em seus pais. Raia 1: mãe; raia 2: pai; raia 3: paciente; raia 4: heterozigoto-controle; raia 5: não-digerido; raia 6: água Milli Q; eaia 7: marcador de peso molecular

CT: Colesterol total; HDL: lipoproteína de alta densidade; LDL: lipoproteína de baixa densidade; TC: triglicerídeos; $\mathrm{G}$ : glicose.

\section{Discussão}

HA é reconhecida como um dos maiores fatores de risco para o desenvolvimento de aterosclerose e doença coronariana. Frequentemente, coexistem hipertensão e dislipidemia, assim como DM2, aumentando fortemente o risco da doença aterosclerótica. A manutenção dos níveis ideais de lipídios constitui um dos objetivos do tratamento dos pacientes diabéticos, justamente para evitar a progressão das complicações macrovasculares secundárias ao diabetes ${ }^{(15)}$. A terapia anti-hipertensiva no paciente diabético é baseada normalmente em estudos realizados em hipertensos somente e estes podem não sofrer a interferência metabólica das drogas hipoglicemiantes. A avaliação dos efeitos adversos 
dos anti-hipertensivos, mesmo havendo o uso de hipoglicemiantes, deve ser considerada como os efeitos metabólicos de certas drogas anti-hipertensivas no perfil lipídico que podem aumentar o risco coronariano, mesmo produzindo uma adequada redução da pressão sanguínea.

Inicialmente, em nosso estudo, compararam-se grupos de pacientes hipertensos (grupo A) com pacientes diabéticos hipertensos tratados com várias formas de terapia, captopril somente (grupo B), captopril combinado com hidroclorotiazida (grupo C), captopril associado com propranolol ou metildopa ou nifedipina (grupo D) e, por último, grupo de pacientes sem captopril, utilizando antagonista do receptor de Angiotensina II somente ou associado a propranolol ou nifedipina (grupo E). Os resultados encontrados do perfil lipídico não demonstraram diferenças estatísticas significativas entre os grupos, no entanto, é importante ressaltar que os valores médios de colesterol total estão dentro dos limites desejáveis e HDL também dentro de padrões para pacientes diabéticos. Porém, os valores de LDL deveriam ser entre os grupos de pacientes diabéticos menores que $100 \mathrm{mg} / \mathrm{dl}$ e triglicerídeos menores que $150 \mathrm{mg} / \mathrm{dl}$, garantindo menor risco. Os pacientes do grupo $\mathrm{C}$, tratados com captopril combinado com hidroclorotiazida, apresentaram os menores níveis de colesterol total e triglicerídeos e os maiores níveis de HDL colesterol, semelhante aos valores encontrados nos pacientes do grupo $\mathrm{A}$. Mesmo não havendo significância estatística, os níveis de HDL maiores que $45 \mathrm{mg} / \mathrm{dl}$ são satisfatórios, associados à proteção para doenças coronarianas. Maranhão, em 2004(1), estudando pacientes hipertensos e hipercolesterolêmicos, observou que o uso do captopril não provoca alteração na cinética dos lipídios no sangue, não altera os valores de TG, HDL, evidenciando que este agente terapêutico não é deletério ao perfil lipídico, e além disso observou redução dos níveis de LDL, efeito antiaterogênico.
Karim et al., em 2001 ${ }^{(14)}$, estudaram o efeito combinado de captopril e diurético tiazídico no perfil lipídico de muIheres hipertensas e encontraram níveis de triglicerídeos, HDL e LDL significativamente diferentes do controle, porém com resultados médios semelhantes a este trabalho, com aumento dos níveis de HDL e redução dos níveis de LDL. Karim et al. concluem que a combinação é benéfica para o perfil lipídico e sugerem a necessidade de estudos com um número maior de pacientes e de ambos os sexos. Cifková et al., em 2000(5) também estudaram o perfil lipídico de 80 pacientes hipertensos tratados com duas combinações diferentes, um grupo com bloqueador de canal de cálcio e captopril e outro captopril e hidroclorotiazida, e também não observaram diferenças no perfil lipídico e os níveis de HDL foram maiores significativamente nos pacientes tratados com captopril e bloqueador de canal de cálcio.

Holzgreve et al., em 1997(17), relataram também que a combinação da hidroclorotiazida com captopril não tem efeito adverso sobre o metabolismo glicídico e lipídico, mas questiona se o captopril, inibidor da ECA, tem a completa capacidade de conter os efeitos metabólicos adversos do diurético, uma vez que existem drogas hipoglicemiantes sendo associadas a estes pacientes. No nosso estudo, o fato de não haver diferenças significativas no perfil lipídico entre os pacientes hipertensos e diabéticos em uso de captopril somente e os associados com hidroclorotiazida nos leva a crer que o efeito deletério aterogênico provocado pelo uso da hidroclorotiazida ${ }^{(1,19,21)}$ é minimizado pela associação com o captopril, devido aos níveis maiores de HDL e triglicerídeos menores, mesmo naqueles pacientes diabéticos não-controlados, em que a glicação induzida pela hiperglicemia poderia diminuir os níveis de $\mathrm{HDL}^{(18)}$, diminuindo seu efeito protetor. No entanto, o que observamos é um resultado de HDL semelhante ao do paciente com controle metabólico satisfatório, indicando-nos que a associação tem efeito protetor, diminuindo o risco ateroesclerótico desses pacientes.

\section{Referências}

1. ALVES, R. J. et al. Ausência de efeito do captopril no metabolismo de uma emulsão lipídica artificial semelhante aos quilomícrons em pacientes hipertensos e hipercolesterolêmicos. Arq Bras Card, v. 83, n. 6 , p. 508-11, 2004.

2. AMERICAN DIABETES ASSOCIATION. Clinical practice recommendations. report of the expert committee on the diagnosis and classification of diabetes mellitus. Diabetes Care, v. 26, suppl. 1, p. S5-20, 2003.
3. BEM, A. F.; KUNDE, J. A importância da determinação da hemoglobina glicada no monitoramento das complicações crônicas do diabetes mellitus. J. Bras. Patol. Med. Lab., v. 42, n. 3, p. 185-91, 2006.

4. CHOBANIAN, A. V. etal. National high blood pressure education program coordinating committee. The seventh report of the joint national committee on prevention, detection evaluation, and treatment of high blood pressure: The JNC 7 report. JAMA, v. 289, n. 19, p. 2560-72, 2003. 
5. CIFKOVÁ, R. et al. Evaluation of the effects of fixed combinations of sustained-release verapamil/trandolapril versus captopril/hydrochlorothiazide on metabolic and electrolyte parameters in patients with essential hypertension. Journal of Human Hypertension, v. 14, p. 347-54, 2000.

6. DCCT RESEARCH GROUP. iabetes control and complications trial (DCCT). The effect of intensive treatment of diabetes on the development and progression of long-term complications in insulin-dependent diabetes mellitus. N Engl J Med., v. 329, p. 977-86, 1993.

7. DEFRONZO, R. A. Pathogenesis of type 2 (non-insulindependent) diabetes mellitus: a balanced overview. Diabetologia, v.35, p.389-97, 1992.

8. DODSON, P. M. Hypertension and diabetes. Curr Med Res Opin, v. 18, n. 1, p. 48-57, 2002.

9. FERRANNINI, E. et al. Insulin resistance in essential hypertension. N. Engl. J. Med., v. 317, p. 350-57, 1987.

10. FERRARA, L. A. et al. Doxazosin and captopril in mildly hyper-cholesterolemic hypertensive patients. The Doxazosin-Captopril in Hypercholeste-rolemic Hypertensive Study. Hypertension, v. 21, p. $97-$ 104, 1993.

11. FOLLOW-UP Report on the Diagnosis of Diabetes Mellitus. The Expert Committee on the Diagnosis and Classification of Diabetes Mellitus. Diabetes Care, v. 26, p. 3160-7, 2003.

12. FULLER, J. H. Epidemiolgy of hypertension associated with diabetes mellitus. Hypertension, v. 7, p. 3-7, 1985.

13. HUNTER, S. J. Comparison of effects of catopril used either alone in combination with a thiazide diuretic on insulin action in hypertensive type 2 diabetic patients: a double-blind crossover study. Diabetic Medicine, v. 16, p. 482-87, 1999.

14. KARIM, S.; BEGUM, A.; JAVED, N. Combined effect of captopril and thiazide diuretic on serum lipid profile in hypertensive women. Journal of Ayub Medical College: JAMC, v. 13, n. 1, p. 19-21, 2001.

15. LIMA, L. M. et al. Correlação entre os níveis plasmáticos de apolipoproteínas A-I e B e o perfil lipídico em indivíduos com e sem diabetes mellitus tipo 2 e hipertensão arterial. J. Bras. Patol. Med. Lab., v. 41, n. 6, p. 41117, 2005.

16. LITHELL, H. ffect of anti-hypertensive drugs on insulin, glucose and lipid metabolism. Diabetes Care, v. 14, p. 203-09, 1991.

17. MIDDEKE, M. et al. The effects of antihypertensive combination therapy on lipid and glucose metabolism: hydrochlothiazide plus sotalol vs. hydrochlorothiazide plus captopril. International Journal of Clinical Pharmacology and Therapeutics Int J Clin Pharmacol Ther, v. 35, n. 6, p. 231-4, 1997.

18. NAKHJAVANI, M. et al. HbA1c negatively correlates with LCAT activity in type 2 diabetes. Diabetes Res Clin Pract, v. 81, n. 1, p. 38-41, 2008.

19. POLLARE, T. et al. A comparison of the effects of hydrochlorothiazide and captopril on glucose and lipid metabolism in patients with hypertension. $N$. Engl. J. Med., v. 321, p. 868-73, 1989.

20. POSICIONAMENTO OFICIAL 2004. A importância da hemoglobina glicada (A1C) para a avaliação do controle glicêmico em pacientes com diabetes mellitus: aspectos clínicos e laboratoriais. Disponível em: http://www.sbpc.org.br. Acessado em: 15 mar 2008.

21. PRINCE, M. J. et al. Metabolic effects of hydrochlorothiazide and enalapril during treatment of hypertensive diabetic patient. Arch. Intern. Med., v. 148, p. 2363-8, 1988.

22. UK PROSPECTIVE DIABETES STUDY GROUP (UKPDS). Intensive blood glucose control with sulphonylureas or insulin compared with conventional treatment and risk of complications in patients with type 2 diabetes. Lancet; v. 352, p. 837-53, 1998.

23. V DIRETRIZES Brasileiras de Hipertensão. Disponível em: www.sbn.org.br/Diretrizes/ VDiretrizesBrasileirasdeHipertensãoArterial.pdf. Acessado em: 03 nov 2008.

24. WHO (WORLD HEALTH ORGANIZATION). Diabetes mellitus. Fact sheets, n. 138, abr 2002. Disponível em: http://www.who.int/inf-fs/en/fact138.html. Acessado em: 27 set 2004. 